\title{
Impact of Pharmacists' Counseling in Patients' Knowledge of Warfarin in a Tertiary Care Cardiac Centre: A Prospective Randomized Controlled Study
}

\author{
Sweta Shrestha*, Basudev Bhattarai, Avisek Ghimire, Yogesh Karmacharya, Saugat Khanal, Suman \\ Shahukhal
}

Department of Pharmacy, Kathmandu University, Dhulikhel, NEPAL.

\begin{abstract}
Background: Warfarin is the mainstay of anticoagulation therapy; however, its' use has been associated with many complications especially bleeding. Adequate patients' knowledge on warfarin can improve anticoagulation control with reduction in adverse drug reactions and other associated complications. The objective of the study was to assess the impact of pharmacist's counseling on patient's knowledge of warfarin. Methods: A prospective randomized controlled study was carried out in Shahid Gangalal National Heart Center, Kathmandu, Nepal. A simple random sampling technique was used to enroll the participants in the intervention and control groups. A total of 99 patients participated in the study. Both the groups were administered the same questionnaire to assess their baseline knowledge. Verbal counseling of 20 min was provided to the intervention group whereas the control group received no pharmacist intervention. Final assessment of the knowledge level of both groups was done after a period of 2 months using the same questionnaire. Results: A significant increment in percentage of patients with adequate knowledge on warfarin was found in the counseled group after intervention $(p<0.05)$. The results further suggested that significantly increased proportion of patients in the intervention group achieved a better control of INR (International Normalized Ratio) after the intervention. No significant association was found between duration of therapy, gender, age and the knowledge score of the patients. Conclusion: Pharmacist provided counseling can significantly improve patients' knowledge on warfarin.

Key words: Anticoagulation, Counseling, INR, Knowledge, Warfarin.
\end{abstract}

\section{INTRODUCTION}

Warfarin is the most widely recommended oral anticoagulant, used in the prevention of clotting among patients at risk for stroke or thromboembolic events. ${ }^{1}$ The use of warfarin is challenged by its complex pharmacology and intrinsic risk of adverse outcomes, which requires routine INR (International Normalized ratio) monitoring to ensure safe and effective therapy. ${ }^{2,3}$ Clinicians have previously cited an unwillingness to prescribe warfarin due to its interactions with diet, drugs and need for monitoring, all of which relies on patient compliance and treatment instructions and therefore is dependent on effective patient education. ${ }^{4}$

Warfarin is a major contributor to potentially preventable drug-related adverse events, partly due to inadequate patient knowledge. ${ }^{5}$
A validated set of questionnaire provides a simple method of determining patients' knowledge of anticoagulation. ${ }^{6}$ Studies have concluded that patients' warfarin knowledge, a major contributor to anticoagulation control is poor and more attention should be given in educating the patients' on warfarin use. ${ }^{7}$ Hence, it is of paramount importance for patients to have proper knowledge about the factors that may lead to warfarin related complications. Various studies have found that pharmacist provided verbal counseling improves warfarin knowledge. ${ }^{4,8,9}$ This can be accomplished by counseling so as to help prevent adverse health outcomes. ${ }^{4}$ However, studies assessing impact of educational intervention among Nepalese population is limited. Therefore, the study aims to assess the impact of pharmacist's counseling in
DOI: 10.5530/ijopp.13.2.26

Address for correspondence: Sweta Shrestha, Department of Pharmacy, Kathmandu University, Dhulikhel, Kavre, NEPAL.

Phone no: +977-9841470887

Email Id: swetashres@gmail.com

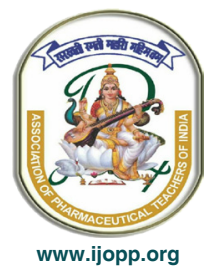


patient's knowledge of warfarin therapy.

\section{MATERIALS AND METHODS Study site and setting}

The study was conducted in the outpatient department of Shahid Gangalal National Heart Center, Kathmandu. This is a 200 bedded tertiary care hospital with an outpatient flow of 350 patients per day.

\section{Study Duration}

The study was conducted for duration of 7 months from March to September 2015.

\section{Selection of Participants}

Simple random sampling technique was used to assign the patients either to the intervention (pharmacist counseling) or the control group (usual care) where they had an equal chance of being allocated to either of the two groups. The patients selected in both the groups were similar in terms of socio-demographic variables. This ensured avoidance of patient selection bias in the study. The two homogenous groups were interviewed separately in different rooms and were kept unaware of their subjection to either intervention or control category to warrant achievement of blinding in the study. A patient who did not receive the pharmacist counseling but were similar to the intervention group in respect to other variables was the control group. All the patients who met the inclusion criteria and visited the outpatient pharmacy during the study duration were enrolled in the study. A total of 99 patients $(n=53$, intervention group versus $n=46$, control group) were interviewed and 29 patients were lost to follow up.

\section{Inclusion Criteria}

- Patients visiting the outpatient pharmacy of Shahid Gangalal National Heart Center aged over 15 years.

- Patients who had been taking warfarin for at least 1 month.

- Patients willing to participate in the study.

\section{Exclusion Criteria}

- Patients who were recent users of warfarin (Less than 1 month).

- Patients admitted in the hospital.

\section{Research Tool}

The study was conducted using a questionnaire comprising of 15 questions on knowledge assessment, adapted from the pre-validated AKA (Anticoagulant Knowledge Assessment) Questionnaire and OAK (Oral Anticoagulant Knowledge) Questionnaire. The questionnaire was validated by pretesting. A pilot study was performed on 10 percent of the total expected sample size to strengthen the reliability and validity of the research. Each correct answer scored 1 point whereas an incorrect answer scored zero point. As a counseling aid, an information leaflet on warfarin therapy was provided to the patients in the intervention group. The information leaflet was compiled from "Oral Anticoagulant Therapy - Important information for patients" published by The National Patient Safety Agency, London and "The patient information, Warfarin Guide for patient and families" published by the John Hopkins Hospital. The information included in the leaflet was translated into Nepali language which was checked and approved by an expert.

\section{Study Procedure}

A prospective randomized controlled study was conducted over a period of 7 months to assess the effect of pharmacist provided counseling in the knowledge of patients on warfarin. The study was approved by the ethical review board in Shahid Gangalal National Heart Center. The questionnaire was administered to the participants by the researcher himself. In the control group, the pharmacist did not intervene and only documented the baseline knowledge on warfarin therapy, on the first visit as well as on the subsequent visit after 2 months. In the intervention group the pharmacist obtained the baseline data and provided counseling to the patients for $20 \mathrm{~min}$ on warfarin therapy and its significance, dietary discipline, commonly observed adverse drug reactions and related preventive measures, significance of INR monitoring and warfarin drug interactions. A pharmacist prepared, leaflet containing necessary information on warfarin was also provided to the patients in the intervention group. After 2 months, the same questionnaire was used to assess the effect of counseling in the knowledge level of the patients who had received the educational intervention. In addition, INR (International Normalized Ratio) value in both the groups was collected at two time points, on the day of first interview and after 2 months. Twenty-nine patients were lost to follow up. Follow up data of only 36 patients in the intervention group and 34 patients in the control group could be obtained. Demographic details such as patients' age, gender, duration of warfarin therapy, INR value on the day of first interview and other clinically relevant information such as indication of warfarin therapy was also collected in the patient performa. A 


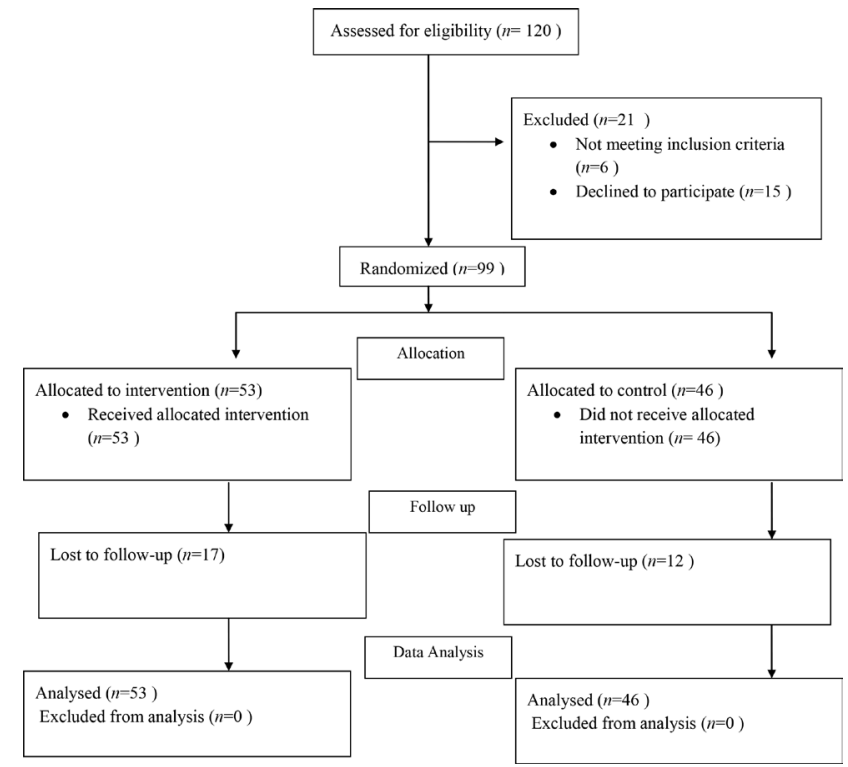

Figure 1: Consort flow diagram of the phases of a randomized controlled study (enrolment, intervention, allocation, follow up and data analysis).

comparison of the number of patients with adequate knowledge in the intervention and the control group was done. Furthermore, a comparison of the number of patients with INR within the target goal was done, pre and post-intervention in the counseled group. Similarly, the difference in the number of patients with their INR value within the target range was also noted in the control group after 2 months. Disparity between percentages of patients answering each question correctly in the intervention and the control group was also observed. The data collected were entered in a separate spreadsheet of EXCEL and was analyzed using Statistical package for social sciences (spss) software version 20. (Figure 1)

\section{Statistical Analysis}

\section{Application of the binomial distribution}

The questionnaire comprised of 15 questions with four options given as choice $\mathrm{p}=1 / 4$ where, $\mathrm{p}$ is the probability of choosing the correct answer from the four options provided to the interviewee. If $\mathrm{X}$ is the random variable denoting number of correct answers provided by the interviewee, then $\mathrm{X}$ is distributed as a binomial with parameter $n=15$ and $\mathrm{p}=1 / 4$

Then $\mathrm{E}(\mathrm{X})=\mathrm{np}=15(1 / 4)=3.75$ This implies that on average a person answers 4 questions correctly even if he/she has no knowledge of the subject. So, if $\mathrm{P}(\mathrm{X} \leq \mathrm{a})$ $=0.95$, the value of $\mathrm{a}=7$ was obtained from the binomial table provided $n=15$ and $\mathrm{p}=1 / 4$ so the $P(\mathrm{X} \geq \mathrm{a})=0.05$.

This implies that if a person answers out of 15,8 or more questions correctly we can conclude with 95\% confidence that the person has adequate knowledge. Impact of pharmacist provided counseling in knowledge level of patients on warfarin was statistically analyzed using the $Z$ test at $95 \%$ confidence interval. Similarly, separate evaluation of difference in number of patients with therapeutic INR after duration of 2 months in the intervention group as well as in the control group was done using the paired $t$-test. $P$ value $<0.05$ was considered to be statistically significant. Chi-square test was implied to determine the correlation between age, gender, duration of therapy and the knowledge score.

\section{RESULTS}

Table 1 shows the demographic details of the study participants. A larger proportion of the patients were of the age group 31 to 50 years in both the intervention $(40 \%, n=21)$ and the control group $(39 \%, n=18)$. Counseled and the non-counseled group comprised of $64 \%(n=34)$ and $52 \%(n=24)$ of the female patients respectively. Most of the patients in our study had been taking warfarin for duration of more than 5 years.

Table 2 illustrates the distribution of the study population as per the indication of warfarin. Majority of the male patients $(32 \%, n=13)$ in our study had been taking warfarin for DVR (Double Valve Replacement) whereas the majority of the female patients had been taking warfarin for MVR (Mitral Valve Replacement) (36\%, $n=21)$. The most common indication for the use of anticoagulation was found to be MVR $(30 \%, n=30)$.

\section{Evaluation of results of patients with adequate} knowledge on warfarin in the Intervention and

Table 1: Characteristics of study population

\begin{tabular}{ccc} 
Variables & $\begin{array}{c}\text { Intervention Group } \\
\text { (no. (\%) of patients } \\
\mathbf{n = 5 3}\end{array}$ & $\begin{array}{c}\text { Control Group } \\
\text { (no.(\%) of } \\
\text { patients n=46 }\end{array}$ \\
\hline $15-30$ & Age (Years) & \\
$31-50$ & $10(19)$ & $16(35)$ \\
$51-65$ & $21(40)$ & $18(39)$ \\
$65+$ & $17(32)$ & $10(21)$ \\
& $5(9)$ & $2(4)$ \\
Male & Gender & $22(48)$ \\
Female & $19(36)$ & $24(52)$ \\
& $34(64)$ & $8(17)$ \\
$>6$ months & Warfarin duration & $3(7)$ \\
6 months-1 year & $11(21)$ & $13(28)$ \\
1-5 years & $4(8)$ & $22(48)$ \\
$>5$ years & $18(34)$ & \\
\hline
\end{tabular}

Indian Journal of Pharmacy Practice, Vol 13, Issue 2, Apr-Jun, 2020 
Table 2: Distribution of patients as per indication of warfarin

\begin{tabular}{ccc}
\hline Indications & $\begin{array}{c}\text { Male (no.(\%) of } \\
\text { patients) } \mathbf{n = 4 1}\end{array}$ & $\begin{array}{c}\text { Female } \\
\text { (no. (\%) of } \\
\text { patients) } \mathbf{n = 5 8}\end{array}$ \\
\hline Aortic Valve Replacement & $10(22)$ & $8(14)$ \\
Double Valve Replacement & $13(32)$ & $10(17)$ \\
Deep Vein Thrombosis & $3(7)$ & $6(10)$ \\
Mitral Valve Replacement & $9(22)$ & $21(36)$ \\
Tricuspid Valve & & \\
Replacement & $6(15)$ & $13(23)$ \\
\hline
\end{tabular}

Table 3: Evaluation of results of patients with adequate knowledge on warfarin in the intervention and control group

\begin{tabular}{cc}
$\begin{array}{c}\text { No. (\%)of patients scoring } \\
\text { more than } 7 \text { in follow up in } \\
\text { control group }(n=34)]\end{array}$ & $\begin{array}{c}\text { No. (\%)of patients scoring } \\
\text { more than } 7 \text { after counseling } \\
\text { in Interventional group } \\
(\mathbf{n}=36)]\end{array}$ \\
\hline $13(38)$ & $32(89)$ \\
\hline
\end{tabular}

Table 4: Evaluation of results of patients with adequate knowledge on warfarin in intervention group

\begin{tabular}{cc}
$\begin{array}{c}\text { No.(\%) of patients scoring } \\
\text { more than } 7 \text { before } \\
\text { counseling }(n=36)\end{array}$ & $\begin{array}{c}\text { No. }(\%) \text { of patients scoring } \\
\text { more than } 7 \text { after counseling } \\
(n=36)\end{array}$ \\
\hline $8(22)$ & $32(89)$ \\
\hline
\end{tabular}

\section{control group}

Table 3 shows a comparison of the number of patients with adequate knowledge on warfarin therapy in the intervention $(38 \%, n=13)$ and the control group $(89 \%$, $n=32$ ). A significant improvement in warfarin knowledge was seen in the intervention group than in the control group $(p<0.05)$

\section{Evaluation of results of patients with adequate knowledge on warfarin in intervention Group}

Table 4 shows the number of patients with adequate knowledge on warfarin therapy before counseling $(22 \%, n=8)$, which rose to a figure of $(89 \%, n=32)$ after counseling. A significant increment in the number was observed 2 months after counseling $(p<0.05)$.

Table 5 illustrates the comparison of percentage of patients giving correct answers between the intervention and the control groups. It is representative of the data collected 2 months after the counseling. Majority of the questions were answered correctly in the counseled group when compared to the non-counseled group. Exception was the question related to interaction with alcohol (Question 5) and question related to interaction with vitamin (Question 9) where the non- counseled group achieved a slightly higher score than the counseled

Table 5: Percentage of patients giving correct answers in intervention and control group

\begin{tabular}{|c|c|c|}
\hline Questions & $\begin{array}{l}\text { Counseled No.(\%) of patients } \\
\qquad(n=36)\end{array}$ & $\begin{array}{l}\text { Non-counseled No.(\%) of patients } \\
\qquad(n=34)\end{array}$ \\
\hline Identifying different strengths of warfarin & $18(50)$ & $17(50)$ \\
\hline Action in missing a dose & $35(97)$ & $31(91)$ \\
\hline Food interaction & $34(94)$ & $30(88)$ \\
\hline PT-INR means & $35(97)$ & $28(82)$ \\
\hline Interaction with alcohol & $12(33)$ & $12(35)$ \\
\hline Factor affecting warfarin action & $18(50)$ & $48(14)$ \\
\hline Condition requiring emergency unit visit & $27(75)$ & $15(44)$ \\
\hline Medicine of choice for headache relief & $29(80)$ & $24(70)$ \\
\hline Interaction with vitamin & $12(33)$ & $14(41)$ \\
\hline Fact about spinach intake & $36(100)$ & $33(97)$ \\
\hline Most likely adverse effect of warfarin & $97(27)$ & $2(5)$ \\
\hline When to take warfarin & $36(100)$ & $34(100)$ \\
\hline Effect seen when INR is high & $58(16)$ & $48(14)$ \\
\hline Effect necessitating to call Coumadin clinic & $17(47)$ & $58(17)$ \\
\hline Length of time warfarin should be continued & $36(100)$ & $34(99)$ \\
\hline
\end{tabular}


group. All of the patients both in the counseled and the non-counseled group knew the best time of the day to take warfarin.

Table 6 displays the percentage of patients answering each question correctly before and after the counseling. The results showed an increase in the percentage of patients answering each question correctly post counseling. Furthermore, the results illustrated that a high percentage $(70 \%, n=37)$ of the patients had less idea of identifying the different strengths of warfarin tablets (Question no.1). Knowledge concerning the administration time (Question no. 12) was also assessed. The proportion of patients who knew the correct administration time was almost $(94 \%, n=50)$ even before counseling which rose to a perfect $(100 \%, n=53)$ after pharmacist's counseling. Only $(25 \%, n=13)$ of the patients knew about the action to be taken after missing a dose (Question no. 2) but the proportion increased to a whopping ( $97 \%, n=35$ ) after counseling. An increase in mean knowledge score from 6.58 to 10.05 was observed after the intervention. The patient's knowledge was assessed on the following criteria: importance of INR monitoring, warfarin-food interactions, warfarin drugs interactions, adverse events of warfarin and actions to be taken when they occur and precautions to be taken while on warfarin therapy.

\section{Correlation between age, gender, duration of warfarin therapy and knowledge}

No significant association was found between age, gender, duration of warfarin therapy and the knowledge level of the patients $(p>0.05)$

\section{Assessment of the impact of pharmacist counseling on control of INR}

Comparison of the number of patients with INR within therapeutic range between the first day of interview and after 2 months, in the intervention and control groups is provided in Table 7 and Table 8 respectively. A significant increase in number of patients with INR within target range was obtained using paired $t$-test at $95 \%$ confidence interval in the intervention group $(n=30, p=0.005)$ whereas no significant change was observed in the control group $(n=25 ; p=0.083)$.

\section{DISCUSSION}

The study shows that majority of the warfarin users in both the intervention and control groups were of the

Table 6: Percentage of patients correctly answering in intervention group

\begin{tabular}{|c|c|c|}
\hline Questions & $\begin{array}{l}\text { Pre intervention no }(\%) \\
\text { of patients }(n=53)\end{array}$ & $\begin{array}{l}\text { Post intervention no(\%) } \\
\text { of Patients }(n=36)\end{array}$ \\
\hline Identifying different strengths of warfarin & $16(30)$ & $18(50)$ \\
\hline Action in missing a dose & $13(25)$ & $35(97)$ \\
\hline Food interaction & $16(30)$ & $34(94)$ \\
\hline PT-INR means & $31(58)$ & $35(97)$ \\
\hline Interaction with alcohol & $7(13)$ & $12(33)$ \\
\hline Factor affecting warfarin action & $20(38)$ & $18(50)$ \\
\hline Condition requiring emergency unit visit & $32(61)$ & $27(75)$ \\
\hline Medicine of choice for headache relief & $28(52)$ & $29(80)$ \\
\hline Interaction with vitamin & $7(13)$ & $12(33)$ \\
\hline Fact about spinach intake & $47(88)$ & $36(100)$ \\
\hline Most likely adverse effect of warfarin & $3(5)$ & $97(27)$ \\
\hline When to take warfarin & $50(94)$ & $36(100)$ \\
\hline Effect seen when INR is high & $4(8)$ & $58(16)$ \\
\hline Effect neccesitating to call Coumadin clinic & $8(16)$ & $17(47)$ \\
\hline Length of time warfarin should be continued & $50(94)$ & $36(100)$ \\
\hline
\end{tabular}

Table 7: INR control comparison in intervention group

No. (\%)of patients with INR in therapeutic range pre- counseling, $(n=30)$

No. of patients with INR in therapeutic range post-counseling, $(n=30)$
$12(40)$

24(80)
Table 8. INR control comparison in control group

$\begin{gathered}\text { No. (\%)of patients with INR } \\ \text { in therapeutic range on first } \\ \text { visit }(n=25)\end{gathered}$
$12(48)$

No. (\%)of patients with INR in therapeutic range on follow up ( $n=25)$ $17(68)$ 
age group 31-50 years (40\% vs 39\%). Percentage of female patients using warfarin was higher than their male counterparts in both the categories $(64 \%$, intervention group and $52 \%$, control group). A larger proportion of patients had been under warfarin therapy for more than 5 years. (Table 1) The study revealed the most common indication for warfarin among male patients was DVR whereas it was MVR among the female patients $(32 \%$ and $36 \%$ ) respectively. (Table 2 )

Pharmacist provided counseling can serve as a strong supportive measure to enhance warfarin related knowledge among the patients ${ }^{8,9}$ which further can serve as a major contributor to anticoagulation control. ${ }^{7}$ The results of this research also adheres to the above finding. This study examined whether pharmacist provided counseling improved the knowledge of patients regarding their warfarin therapy. Only $(38 \%$, $n=13)$ of patients in the control group was found to have adequate knowledge on warfarin against a good number of $(89 \%, n=32)$ in the intervention group. Table 3 . Quite interestingly, a strikingly significant increase in percentage of patients with adequate knowledge was observed in the intervention group, 2 months post counseling $(22 \%, n=8$ vs. $89 \% ; n=32)$. Table 4 . This is consistent with the findings by Lakshmi et al. where mean knowledge score of patients' on anticoagulation rose significantly from $5.6(\mathrm{SD}=3.2)$ to $13.8(\mathrm{SD}=0.94)$ $(P=0.000)$ after receiving counseling from clinical pharmacist, whereas no significant enhancement was observed in the control group $(\mathrm{p}=0.218) .{ }^{9}$ Another study by Collins et al. is reflective of similar findings where an increment of $34.9 \%$ in terms of number of patients achieving a passing score was noted after receiving verbal counseling on warfarin. ${ }^{8}$ But it is not in line with the result acquired by Hassan et al. which showed no significant differences in the knowledge of warfarin between the counseled and the non-counseled groups. However, the result by Hassan et al. is based on only 3 knowledge categories namely mechanism of action, interactions between alcohol and warfarin, side effects of warfarin. The reason for this difference probably is due to inclusion of 15 different knowledge assessment criteria in the present study which might have possibly produced a result contrary to Hassan $e t a l .^{10}$ A positive impact of pharmacist provided counseling has also been demonstrated by the fact that most of the questions had been correctly addressed by patients in the intervention group as compared to those in the control group. (Table 5)

Number of patients with INR within therapeutic range rose significantly in the intervention group $(n=30$, $p=0.005)$ whereas no significant alteration was observed in the control group $(n=25 ; p=0.083)$. (Table 7,8$)$. This corroborates the findings of Khan et al. ${ }^{11}$ who observed the percentage of time spent within the therapeutic range between two intervention groups and one control group. Although, no statistically significant difference was seen but the increase in percentage of time spent within the therapeutic range was relatively higher in the education plus self-monitoring group and the education alone group with a mean difference of 14.1 and 8.8 respectively, following 6 months of intervention. The findings of the present study backs the result of Khudair et al. ${ }^{12}$ where significantly higher proportion of patients receiving warfarin education had controlled INR than the patients who did not receive any educational intervention (48\%, $n=116$ vs. $26 \%, n=24 ; P=0.0136)$. Lakshmi et al. ${ }^{9}$ has findings similar to ours where percentage of INRs within therapeutic range was found to be significantly greater in the intervention group than in the control group $(77.4 \%$ vs. $46.5 \% ; P=0.000)$.

In regard to patients' comprehension of interaction of warfarin with food, initially $(70 \% n=37)$ of patients were unaware of the foods that interfered with the working of warfarin. This number; however, reduced to $(6 \%, n=2)$ two months after counseling. Approximate figures have been presented in two of the previous studies. ${ }^{4,13} \mathrm{~A}$ great majority of patients $(87 \%, n=46)$ believed in absence of interaction between alcohol and warfarin, which fortunately declined to $(67 \%, n=24)$ after counseling. Yahaya et al..$^{14}$ and Shrestha et al. ${ }^{13}$ reportedly presented a statistic of $34.6 \%$ and $44.1 \%$ respectively, of those who were ignorant of this potential interaction. Similarly, $48 \%$ of patients failed to select the safest non-steroidal anti-inflammatory drug while on warfarin, which after counseling lowered to $20 \%$. A previous study reported a Figure of $(44.1 \%, n=34)$ approximating to our data. ${ }^{13}$ Patients' ignorance on these essential factors may be ascribed to lack of adequate educational and assessment sessions in the hospitals. Nevertheless, proper counseling by pharmacists can alleviate the misconception and ignorance of patients regarding warfarin therapy.

No correlation was established between gender of the patients and warfarin knowledge. This is underpinned by the findings of previous studies. ${ }^{10,12-15}$ Similarly, warfarin knowledge was found to be unaffected by age. This is in contrary to the findings where age was found be negatively correlated with total knowledge score of the patients. ${ }^{47,10,15,16}$ However, a study by Shrestha et al. ${ }^{13}$ has findings similar to ours which found no association between age of the patients and warfarin knowledge. Furthermore, duration of therapy was also found to be uncorrelated with patient's knowledge which is similar to the results obtained by Baker et al. ${ }^{17}$ and Hasan et al. ${ }^{11}$ but opposes the findings of Shrestha $\mathrm{S}$ et al. ${ }^{13}$ 


\section{What this study adds to existing evidence?}

This study is first of its kind to address the impact of pharmacist provided counseling in knowledge of warfarin as well as its aid in achieving INR control among the Nepalese patients. Findings of this study support the need and benefits of introducing the policies for physician pharmacist collaboration anticoagulation services in developing countries like Nepal.

\section{Study Limitation}

Our study had a number of limitations. Firstly, this was a single institutional study and $29 \%$ of patients were lost to follow up. Secondly due to limited time frame we could provide only one session of counseling. Separate studies have suggested that no correlation exist between the patients' knowledge of warfarin therapy and anticoagulation control. ${ }^{15,18}$ However, the present study did not look into this matter. Although, the current study adds to the existing finding that educational intervention improves INR control, this result demands further investigation since only a single post INR value after 2 months of intervention was taken into account to assess the effect of counseling on INR. Additionally, INR values of only 30 patients in the intervention group and only 25 patients in the control group could be obtained at two time points.

\section{Future Research Direction}

We realize that this subject merit further detailed exploration of impact of educational intervention and self-monitoring on INR control of patients on warfarin due to the aforementioned limitations.

\section{CONCLUSION}

Patients' who received pharmacists' counseling had better knowledge on warfarin therapy than those who did not receive the counseling. Our findings conclude that pharmacists' counseling aided by informational leaflets can play an important role in improving patients' warfarin related knowledge and probably have a remarkably positive impact on the control of INR as well.

\section{ACKNOWLEDGEMENT}

The authors are very much grateful to Shahid Gangalal National Heart Centre for granting the permission to conduct the study. We would like to pay our sincere gratitude to Dr. Rajani Shakya, Acting Head of Department, Department of Pharmacy, Kathmandu University; Dr. Sujeeb Rajbhandari, Research Head, Shahid Gangalal National Health Center; Professor
Dr. Jyoti Upadhaya, Head of Department, Department of Natural Science for their help in conducting the study.

\section{CONFLICT OF INTEREST}

The authors declare no conflict interest.

\section{Ethical approval}

The study was approved by the ethical review board in Shahid Gangalal National Heart Center. The approval (reference) number provided for this research was 1458. Prior to the collection of the data, the participants were informed about the purpose and objective of the study and consent was obtained from each of them. The identity of the participant was kept confidential.

\section{ABBREVIATIONS}

AKA: Anticoagulant Knowledge Assessment; AVR: Aortic Valve Replacement; DVR: Double Valve Replacement; DVT: Deep Vein Thrombosis; INR: International Normalized ratio; MVR: Mitral Valve Replacement; OAK: Oral Anticoagulant Knowledge; PT-INR: Prothrombin Time-International Normalized Ratio.

\section{SUMMARY}

Warfarin is a commonly used oral anticoagulant drug associated with the likelihood of adverse events thus necessitating routine INR monitoring. Patients' knowledge on warfarin is believed to be a major contributor to anticoagulation control. Realizing the paucity of information on knowledge of Nepalese patients' on warfarin use and impact of educational intervention on their knowledge level, this study was initiated to address the existing gap. A prospective randomized controlled study was carried out in Shahid Gangalal National Heart Center, Nepal over a period of 7 months. Simple random sampling technique was used to assign the patients either to the intervention (pharmacist counseling) or the control group (usual care). Verbal counseling of 20 min along with an information leaflet was provided to the intervention group whereas the control group received no pharmacist intervention. Assessment of the knowledge level of both groups was done at first meeting and after a period of 2 months using the same questionnaire.

\section{REFERENCES}

1. Cabral KP, Ansell J, Hylek EM. Future directions of stroke prevention in atrial fibrillation: The potential impact of novel anticoagulants and stroke risk stratification. J Thromb Haemost. 2011;9(3):441-9. doi: 10.1111/j.15387836.2010.04179.x

Indian Journal of Pharmacy Practice, Vol 13, Issue 2, Apr-Jun, 2020 
2. Kim JH, Song YB, Shin DH, Kim JS, Choi JO, On YK, et al. How well does the target INR level maintains in warfarin-treated patients with non-valvular a trial Fibrillation. Yonsei Med J. 2009;50(1):83-8. doi:10.3349/ymj.2009.50.1.83

3. Bravata DM, Rosenbeck KR, Kancir S, Brass LM. The use of warfarin in veterans with atrial fibrillation. BMC Cardiovasc Disord. 2004;4(1):18. doi 10.1186/1471-2261-4-18

4. Nasser S, Mullan J, Bajorek B. Challenges of older patients' knowledge about warfare therapy. J Prim Care Community Health. 2012;3(1):56-74. doi: $10.1177 / 2150131911416365$

5. Cheah GM, Martens KH. Coumadin knowledge deficits: Do recently hospitalized patients know how to safely manage the medication?. Home Healthc Nurse. 2003;21(2):94-100.

6. Taylor FC, Ramsey ME, Tan G, Gabbay J, Cohen H. Evaluation of patients' knowledge about anticoagulation treatment. Qual Health Care. 1994;3(2):79-85.

7. Tang EO, Lai CS, Lee KK, Wong RS, Cheng G, Chan TY. Relationship between patients' warfarin knowledge and anticoagulation control. Ann Pharmacother. 2003;37(1):34-9.

8. Collins S, Barber A, Sahm LJ. Pharmacist's counselling improves patient knowledge regarding warfarin, irrespective of health literacy level. Pharmacy. 2014;2(1):114-23. doi: 10.3390/pharmacy2010114

9. Lakshmi R, James E, Kirthivasan R. Study on impact of clinical pharmacist's interventions in the optimal use of oral anticoagulants in stroke patients. Indian J Pharm Sci. 2013;75(1):53-9. doi:10.4103/0250-474X.113550

10. Hasan SS, Shamala R, Syed IA, Basariah N, Chong DW, Mei TK, et al. Factors affecting warfarin-related knowledge and INR control of patients attending physician- and pharmacist-managed anticoagulation clinics. J Pharm Pract. 2011;24(5):485-93. doi: 10.1177/0897190011415684
11. Khan TI, Kamali F, Kesteven P, Avery P, Wynne H. The value of education and self-monitoring in the management of warfarin therapy in older patients with unstable control of anticoagulation. $\mathrm{Br} \mathrm{J}$ Haematol. 2004;126(4):557-64. doi:10.1111/j.13652141.2004.05074.x

12. Khudair IF, Hanssens YI. Evaluation of patients' knowledge on warfarin in outpatient anticoagulation clinics in a teaching hospital in Qatar. Saudi Med J. 2010;31(6):672-7.

13. Shrestha S, Sapkota B, Kumpakha A, Acharya U, Sharma R. Evaluation of patients' knowledge on warfarin in outpatient pharmacy of a tertiary care cardiac center. BMC Res Notes. 2015;8(1):429. doi: 10.1186/s13104-015-1416-1

14. Yahaya AH, Hassali MA, Awaisu A, Shafie AA. Factors associated with warfarin therapy knowledge and anticoagulation control among patients attending a warfarin clinic in Malaysia. JCDR. 2009;3(4):1663-70.

15. Hu A, Chow CM, Dao D, Errett L, Keith M. Factors influencing patient knowledge of warfarin therapy after mechanical heart valve replacement. J Cardiovasc Nurs. 2006;21(3):169-75.

16. Dantas GC, Thompson BV, Manson JA, Tracy CS, Upshur RE. Patients' perspectives on taking warfarin: Qualitative study in family practice. BMC Fam Pract. 2004;5(1):15. doi: 10.1186/1471- 2296-5-15.

17. Baker JW, Pierce KL, Ryals CA. INR Goal attainment and oral anticoagulation knowledge of patients enrolled in an anticoagulation clinic in a Veterans Affairs medical center. J Manag Care Pharm. 2011;17(2):133-42. doi: 10.18553/ jmcp.2011.17.2.13

18. Davis NJ, Billett $\mathrm{HH}$, Cohen HW, Arnsten JH. Impact of adherence, knowledge and quality of life on anticoagulation control. Ann Pharmacother. 2005;39(4):6326. 